\title{
Spin and charge excitations in the correlated multiband metal $\mathrm{Ca}_{3} \mathrm{Ru}_{2} \mathrm{O}_{7}$
}

\author{
J. Bertinshaw,,${ }^{1,2, *}$ M. Krautloher $\odot,{ }^{1}$ H. Suzuki, ${ }^{1}$ H. Takahashi $\odot,{ }^{1}$ A. Ivanov, ${ }^{3}$ H. Yavaş, ${ }^{2, \dagger}$ B. J. Kim,,${ }^{1,4,5}$ \\ H. Gretarsson, ${ }^{1,2, \ddagger}$ and B. Keimer ${ }^{1}$ \\ ${ }^{1}$ Max Planck Institute for Solid State Research, Heisenbergstrasse 1, D-70569 Stuttgart, Germany \\ ${ }^{2}$ Deutsches Elektronen-Synchrotron DESY, Notkestraße 85, D-22607 Hamburg, Germany \\ ${ }^{3}$ Institut Laue-Langevin, 71 Avenue des Martyrs, CS 20156, 38042 Grenoble cedex 9, France \\ ${ }^{4}$ Department of Physics, Pohang University of Science and Technology, Pohang 790-784, South Korea \\ ${ }^{5}$ Center for Artificial Low Dimensional Electronic Systems, Institute for Basic Science (IBS), 77 Cheongam-Ro, Pohang 790-784, South Korea
}

(Received 4 December 2020; accepted 25 January 2021; published 5 February 2021)

\begin{abstract}
We use $\mathrm{Ru} L_{3}$-edge resonant inelastic $\mathrm{x}$-ray scattering to study the full range of excitations in $\mathrm{Ca}_{3} \mathrm{Ru}_{2} \mathrm{O}_{7}$ from meV-scale magnetic dynamics through to the eV-scale interband transitions. This bilayer $4 d$-electron correlated metal expresses a rich phase diagram, displaying long-range magnetic order below $56 \mathrm{~K}$ followed by a concomitant structural, magnetic, and electronic transition at $48 \mathrm{~K}$. In the low-temperature phase, we observe a magnetic excitation with a bandwidth of $\sim 30 \mathrm{meV}$ and a gap of $\sim 8 \mathrm{meV}$ at the zone center, in excellent agreement with inelastic neutron scattering data. The dispersion can be modeled using a Heisenberg Hamiltonian for a bilayer $S=1$ system with single-ion anisotropy terms. At a higher energy loss, $d d$-type excitations show heavy damping in the presence of itinerant electrons, giving rise to a fluorescencelike signal appearing between the $t_{2 g}$ and $e_{g}$ bands. At the same time, we observe a resonance originating from localized $t_{2 g}$ excitations, in analogy to the structurally related Mott insulator $\mathrm{Ca}_{2} \mathrm{RuO}_{4}$. But whereas $\mathrm{Ca}_{2} \mathrm{RuO}_{4}$ shows sharp separate spin-orbit excitations and Hund's-rule driven spin-state transitions, here we identify only a single broad asymmetric feature. These results indicate that local intraionic interactions underlie the correlated physics in $\mathrm{Ca}_{3} \mathrm{Ru}_{2} \mathrm{O}_{7}$, even as the excitations become strongly mixed in the presence of itinerant electrons.
\end{abstract}

DOI: 10.1103/PhysRevB.103.085108

\section{INTRODUCTION}

$\mathrm{Ca}_{3} \mathrm{Ru}_{2} \mathrm{O}_{7}$ is a canonical multiband correlated metal, displaying complex behavior and a rich phase diagram despite the lack of localized $d$-orbital valence electrons generally present in strongly correlated oxides [1-3]. The interactions that drive correlated phenomena away from the Mott state in $4 d$-electron $\mathrm{Ca}_{3} \mathrm{Ru}_{2} \mathrm{O}_{7}$ and other multiband metals such as superconducting $\mathrm{Sr}_{2} \mathrm{RuO}_{4}$ and the iron pnictides are an active and ongoing concern [4]. Vital to this understanding is an investigation of the electronic excitations, which encode information regarding the energy scales of intra- and interionic interaction parameters. Here, resonant inelastic x-ray scattering (RIXS) at the dipole-active $\mathrm{Ru} L_{3}$-edge $(2 p \rightarrow 4 d$ ) has emerged as a key tool, providing a direct momentum and energy-dependent probe of electronic excitations $[5,6]$. Moreover, $\mathrm{Ru} L_{3}$-edge RIXS also covers magnetic transitions,

\footnotetext{
*j.bertinshaw@fkf.mpg.de

${ }^{\dagger}$ Present address: SLAC National Accelerator Laboratory, 2575 Sand Hill Rd, Menlo Park, CA 94025, USA.

*hlynur.gretarsson@desy.de

Published by the American Physical Society under the terms of the Creative Commons Attribution 4.0 International license. Further distribution of this work must maintain attribution to the author(s) and the published article's title, journal citation, and DOI. Open access publication funded by the Max Planck Society.
}

giving detailed insight into spin-wave dynamics [7]. In a recent RIXS study of the single-layered antiferromagnetic Mott insulator $\mathrm{Ca}_{2} \mathrm{RuO}_{4}$, clear spin-orbital and Hund's-rule driven intraionic excitations were identified, from which the spinorbit coupling (SOC) $\xi$, the Hund's-rule energy $J_{\mathrm{H}}$, and the tetragonal crystal-field term $\Delta$ were extracted [5].

The bilayer system $\mathrm{Ca}_{3} \mathrm{Ru}_{2} \mathrm{O}_{7}$ retains a highly anisotropic electric resistivity down to the lowest temperatures [8], where the optical conductivity reveals a small pseudogap of $25 \mathrm{meV}$ [9]. The magnetic properties of the system are dominated by intrabilayer ferromagnetic exchange [10]. Long-range magnetic order forms below $T_{\mathrm{N}}=56 \mathrm{~K}$, where ferromagnetic bilayers stack antiparallel along the $c$ axis (AFM- $a$ ) [11]. At $T_{\mathrm{S}}=48 \mathrm{~K}$, a structural transition that distorts the $\mathrm{RuO}_{6}$ octahedra coincides with an upturn in the out-of-plane resistivity $[1,10]$, and a spin rotation from the $a$ axis to the $b$ axis (AFM$b$ ) that appears to be mediated by an incommensurate spin state $[8,12,13]$. The system also shows a complex magnetic field dependence $[3,14]$ and a remarkable response to doping, where dilute substitution of nonmagnetic $3 d^{0}$-electron $\mathrm{Ti}^{4+}$ on the $\mathrm{Ru}^{4+}$ site quickly pushes the system into a Mott state with antiferromagnetic dynamics characteristic of $\mathrm{Ca}_{2} \mathrm{RuO}_{4}$ $[15,16]$. Taken together, these results signify the presence of a delicate balance of competing interactions, which can be captured in spectroscopic studies at energies beyond the reported small pseudogap.

In this paper, we present a systematic Ru $L_{3}$-edge RIXS study of $\mathrm{Ca}_{3} \mathrm{Ru}_{2} \mathrm{O}_{7}$. Below $T_{\mathrm{S}}$, we capture the dispersion of the in-plane magnon across the entire Brillouin zone, giving 

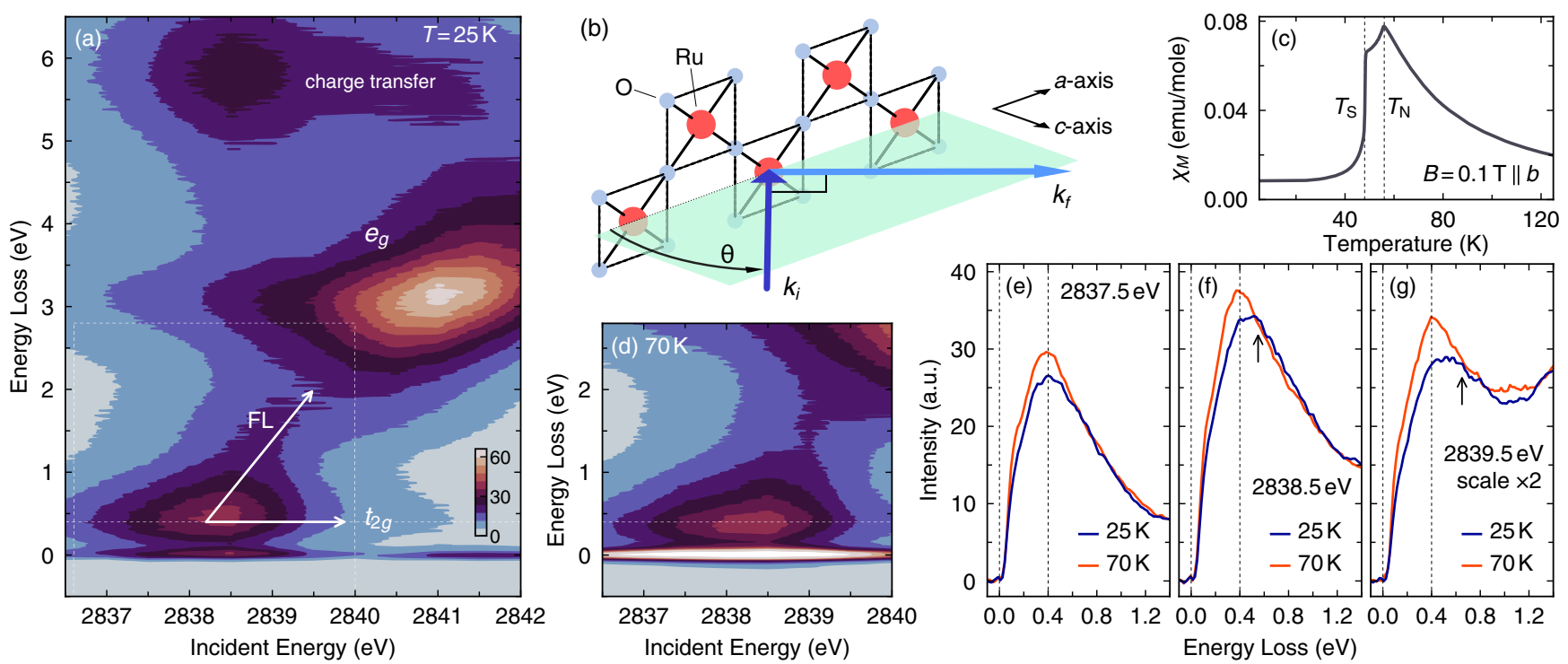

FIG. 1. (a) RIXS energy map taken at $T=25 \mathrm{~K}$ plots the incident-energy dependence of the energy-loss spectra across the Ru $L_{3}$ edge. The quasielastic magnon signal and $t_{2 g}$ feature at $\sim 0.4 \mathrm{eV}$ (horizontal dashed line) resonate around $E_{i}=2838.5 \mathrm{eV}$, while $e_{g}$ excitations around $3 \mathrm{eV}$ strongly resonate at $2841 \mathrm{eV}$. At higher energies $(\sim 6 \mathrm{eV})$, charge transfer excitations are present. (b) The sample was oriented such that the $\mathrm{Ru}-\mathrm{O}-\mathrm{Ru}$ bonds were aligned $45^{\circ}$ to the scattering plane. Outgoing photons $k_{f}$ were detected at a fixed angle of $90^{\circ}$ with respect to the incoming beam $k_{i}$. The momentum transfer was selected by varying the angle $\theta$ between $k_{i}$ and the $\mathrm{RuO}_{2}$ planes. (c) Bulk magnetic response of $\mathrm{Ca}_{3} \mathrm{Ru}_{2} \mathrm{O}_{7}$, with $B=0.1 \mathrm{~T}$ applied along the $b$ axis. (d) The RIXS energy map conducted above $T_{\mathrm{N}}$ [region indicated by a dashed rectangle in (a) ] shows a dramatic enhancement of the quasielastic magnetic excitation and an apparent shift of the $t_{2 g}$ spectral weight to smaller energy loss. (e)-(g) Comparison of individual RIXS spectra from (a) and (d). The quasielastic contribution has been removed. The dashed vertical line at $0.4 \mathrm{eV}$ lies at the $t_{2 g}$ maximum at $T=70 \mathrm{~K}$. The arrows identify a second mode with an independent incident-energy response.

a spin-wave gap of $\sim 8 \mathrm{meV}$, in excellent agreement with inelastic neutron scattering (INS), and a bandwidth of $\sim 30 \mathrm{meV}$. At higher energies, we identify dispersionless $d d$ excitations broadened by itinerant electrons. By studying the RIXS response as a function of incident energy and temperature, we conclude that localized and fluorescencelike excitations are found simultaneously within the $t_{2 g}$ multiplet. A comparison with the structurally related $\mathrm{Ca}_{2} \mathrm{RuO}_{4}$ suggests that the localized modes represent intraionic spin-orbit excitations and Hund's multiplets, which become heavily mixed by the electronic continuum present in $\mathrm{Ca}_{3} \mathrm{Ru}_{2} \mathrm{O}_{7}$.

\section{EXPERIMENTAL DETAILS}

Single crystals of $\mathrm{Ca}_{3} \mathrm{Ru}_{2} \mathrm{O}_{7}$ and $\mathrm{Ca}_{2} \mathrm{RuO}_{4}$ were grown using a floating zone method described previously [17]. Highquality untwinned samples were identified and aligned using polarized light microscopy (see Supplemental Material [18]) and magnetometry. RIXS measurements were carried out at beam line P01 at the PETRA-III synchrotron at DESY, using the IRIXS spectrometer [19]. A cryogenically cooled $\mathrm{Si}(111)$ two-bounce monochromator, secondary $\mathrm{Si}(111)$ fourbounce monochromator (asymmetrically cut), and focusing KB-mirror optics were used in combination with a spherically diced $\mathrm{SiO}_{2}(10 \overline{2})$ analyzer to obtain an overall energy resolution of $\Delta E \sim 75 \mathrm{meV}$ full width at half maximum. To determine the energy of the elastic line, we measured scattering from a droplet of GE varnish applied to the corner of the sample. The RIXS studies were carried out in a $(H 00) \times(00 L)$ scattering geometry (orthorhombic unit cell) as depicted in Fig. 1(b).

\section{RESULTS}

\section{A. Incident-energy dependence}

In Fig. 1(a), a RIXS incident energy $\left(E_{i}\right)$ map of $\mathrm{Ca}_{3} \mathrm{Ru}_{2} \mathrm{O}_{7}$ is shown, collected around the $\mathrm{Ru} L_{3}$-edge $(\sim 2840 \mathrm{eV})$. The spectra are not normalized and only nominal elastic scattering is present, indicative of the high quality of the crystal. The sample was cooled through the magnetically ordered phase to $T=25 \mathrm{~K}<T_{\mathrm{N}}$ and orientated with an incident angle $\theta=45^{\circ}$ such that spectra were collected at the Brillouin zone center $\Gamma$ $\left[Q_{\mathrm{HKL}}=(00 L)\right]$. Following the process described in Ref. [5] for $\mathrm{Ca}_{2} \mathrm{RuO}_{4}$, we consider the spectra as a series of components: a low-energy quasielastic peak followed by electronic $d d$ excitations originating from $t_{2 g}$ states below $1.5 \mathrm{eV}$ and $t_{2 g} \rightarrow e_{g}$ above $2 \mathrm{eV}$. In addition, spectral weight forms at intermediate energies that connects the $t_{2 g}$ and $e_{g}$ features. Since the energy loss of these spectral weights changes with the incident energy, we assign it to a fluorescencelike response. In other words, it originates from delocalized electronic excitations due to the virtually ungapped electron-hole continuum in $\mathrm{Ca}_{3} \mathrm{Ru}_{2} \mathrm{O}_{7}$. This suggests coexisting excitations with Ramanlike (i.e., excitation energy independent of $E_{i}$ ) and fluorescent behavior (i.e., excitation energy following $E_{i}$ ), which has also been reported in recent soft x-ray RIXS experiments on nickel oxides [20], copper oxides [21], and iron arsenides [22].

As charge degrees of freedom in $\mathrm{Ca}_{3} \mathrm{Ru}_{2} \mathrm{O}_{7}$ remain below the energy of the lowest $d d$ excitation, the quasielastic scattering can originate from either spin or charge transitions. In order to identify the origin of the quasielastic scattering, an incident-energy map was therefore repeated in the paramagnetic phase at $T=70 \mathrm{~K}>T_{\mathrm{N}}$, as shown in Fig. 1(d). The 


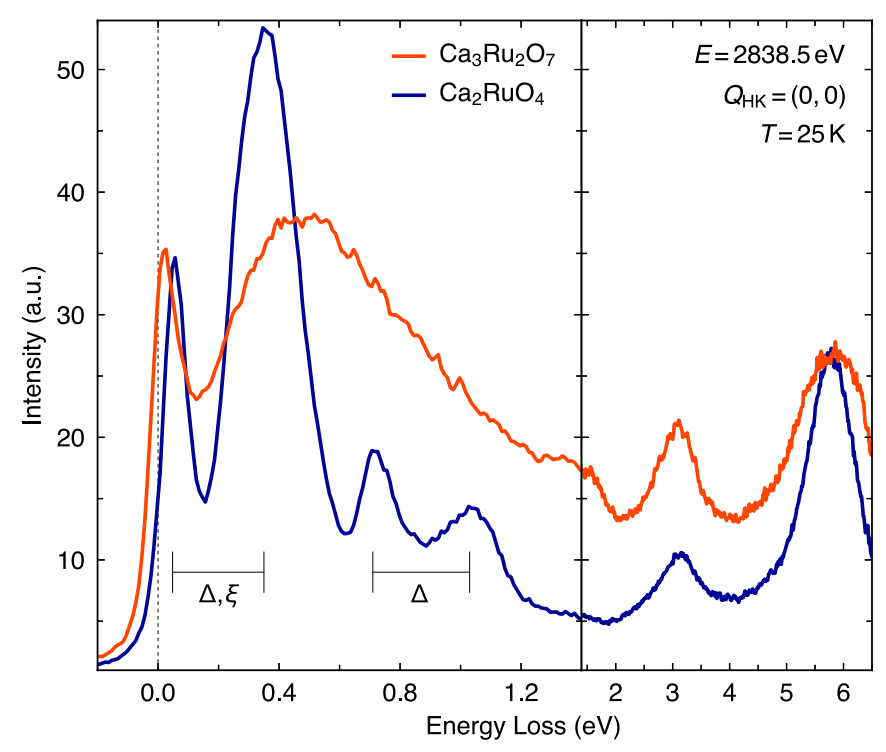

FIG. 2. $\mathrm{Ca}_{3} \mathrm{Ru}_{2} \mathrm{O}_{7}$ RIXS spectrum, measured at $T=25 \mathrm{~K}$ and $E_{i}=2838.5 \mathrm{eV}$ at $Q_{\mathrm{HKL}}=(00 L)\left(\theta=45^{\circ}\right)$, is compared with $\mathrm{Ca}_{2} \mathrm{RuO}_{4}$. The $\mathrm{Ca}_{2} \mathrm{RuO}_{4}$ data is scaled by a factor of 0.7 . Modes associated with spin-wave, $t_{2 g}$, and $e_{g}$ excitations as well as charge transfer are present in both samples. The low-energy splitting of the excitations in $\mathrm{Ca}_{2} \mathrm{RuO}_{4}$ are associated with spin-orbit coupling and tetragonal crystal-field terms.

quasielastic resonance undergoes a dramatic enhancement in the paramagnetic state, even as the intensity of the higherenergy signal remains comparatively unaffected, confirming the magnetic nature of the peak. We note that the intensity remains peaked at $\Gamma$ above $T_{\mathrm{N}}$, indicating that the fluctuations remain ferromagnetic in the paramagnetic regime.

Moving to the $t_{2 g}$ excitation around $0.4 \mathrm{eV}$, marked with horizontal lines in Figs. 1(a) and 1(d), a distinct change in the response is seen as the spectral weight shifts towards lower energies at $T=70 \mathrm{~K}$. This change in the $t_{2 g}$ excitation is made clear in Figs. 1(e)-1(g), where the temperature differences for three incidence energies are compared (the quasielastic magnetic contribution has been subtracted for clarity). Here a complex incident-energy dependence emerges. In the paramagnetic phase, the central weight of the $t_{2 g}$ excitation remains fixed around $0.4 \mathrm{eV}$ [vertical dashed lines in Figs. 1(e)-1(g)], irrespective of $E_{i}$. In the magnetically ordered phase, the overall spectral weight decreases and an energy dependence emerges - spectral weight starts to shift towards higher energies as $E_{i}$ increases [see arrow at $0.6 \mathrm{eV}$ in Figs. 1(f) and $\left.1(\mathrm{~g})\right]$.

\section{B. Comparison with $\mathrm{Ca}_{2} \mathrm{RuO}_{4}$}

The RIXS spectra of $\mathrm{Ca}_{3} \mathrm{Ru}_{2} \mathrm{O}_{7}$ and the structurally related Mott insulator $\mathrm{Ca}_{2} \mathrm{RuO}_{4}$ are compared in Fig. 2. Both systems were measured under the same conditions at the $t_{2 g}$ resonance $E_{i}=2838.5 \mathrm{eV}$ (the $\mathrm{Ca}_{2} \mathrm{RuO}_{4}$ spectrum is scaled by a factor of 0.7 as a visual aid). We note that we are comparing a Mott-insulating system with a correlated "bad" metal with a complex nested Fermi-surface topology [2]. At higher energies, both $\mathrm{Ca}_{3} \mathrm{Ru}_{2} \mathrm{O}_{7}$ and $\mathrm{Ca}_{2} \mathrm{RuO}_{4}$ show a similar signal, with charge transfer type excitations at $6 \mathrm{eV}$ and $t_{2 g} \rightarrow e_{g}$ excitations at $3 \mathrm{eV}$. It is perhaps not surprising that there are no significant changes within this energy regime, given that the overall $\mathrm{RuO}_{6}$ symmetry and $\mathrm{Ru}^{4+}$ and $\mathrm{O}^{2-}$ valencies are identical in the two systems. Below $0.1 \mathrm{eV}$, the magnetic excitation in $\mathrm{Ca}_{2} \mathrm{RuO}_{4}$ is located higher in energy than $\mathrm{Ca}_{3} \mathrm{Ru}_{2} \mathrm{O}_{7}$; a comparison of the spin waves is discussed in the following section.

The largest difference arises within the $t_{2 g}$ regime below $\sim 1.5 \mathrm{eV}$, where the broad asymmetric feature in $\mathrm{Ca}_{3} \mathrm{Ru}_{2} \mathrm{O}_{7}$, which we note has a striking resemblance to $\mathrm{Sr}_{2} \mathrm{RuO}_{4}$ [23], contrasts strongly with the series of sharp excitations observed in $\mathrm{Ca}_{2} \mathrm{RuO}_{4}$. The excitations in $\mathrm{Ca}_{3} \mathrm{Ru}_{2} \mathrm{O}_{7}$ are broadened presumably through coupling with the electronic continuum present at all energies due to the lack of a charge gap. At the same time, the shift in spectral weight to higher-energy loss implies a reconfiguration of the $t_{2 g}$ multiplet structure. The energies of the $t_{2 g}$ modes in $\mathrm{Ca}_{2} \mathrm{RuO}_{4}-\mathrm{a} J=0 \rightarrow 2$ spinorbit excitation at $0.32 \mathrm{eV}$ followed by Hund's-rule driven $S=1 \rightarrow 0$ spin-state transitions around 0.75 and $1.0 \mathrm{eV}$-are controlled primarily by the $\Delta, \xi$, and $J_{\mathrm{H}}$ parameters [5]. In particular, the large splitting between the $S=1 \rightarrow 0$ excitations directly reflects the magnitude of $\Delta$ (see labels in Fig. 2). In $\mathrm{Ca}_{3} \mathrm{Ru}_{2} \mathrm{O}_{7}$, the $\mathrm{RuO}_{6}$ octahedra are less compressed $[10,24]$, which will have the effect of reducing $\Delta$. In the ionic picture, this leads to a smaller splitting of the $S=1 \rightarrow 0$ excitations and lowering of the $J=0 \rightarrow 2$ excitation. As such, the observed dichotomy in the energy-dependency response in Figs. 1(d)-1(f) indicates that underlying the asymmetric $t_{2 g}$ profile are excitations associated with the ionic model, which are heavily mixed with other charge degrees of freedom but remain distinct from the metallic continuum response of the system.

\section{Magnetic dispersion}

The lowest-energy regime associated with magnetic excitations was studied in a detailed $Q$-dependence to follow the spin wave from the zone center, $Q_{\mathrm{HKL}}=(0,0,6.3)$, to slightly beyond the zone boundary $(1.1,0,5.1)$. RIXS spectra in the region close to the elastic line are plotted in Fig. 3(a), which shows the clear dispersion of the spin wave from the zone center to boundary. The intensity is enhanced close to $Q_{\mathrm{H}}=0.0$, consistent with ferromagnetic coupling within the bilayers. The fitted position of the magnon across the Brillouin zone is plotted in Fig. 3(b), which was extracted using a fitting procedure described in the following section. It is overlaid with the low-energy regime of the spin wave determined using inelastic neutron scattering, which was collected using spectrometer IN8, ILL, Grenoble, in the FlatCone configuration (see Supplemental Material [18] and Refs. [26,27]). We note that while the full dispersion could be extracted from the RIXS spectra, the neutron scattering signal became too weak at higher energies to study the spin wave (see, also, Ref. [11]). Despite this, the striking similarity of the system's response to RIXS and INS clearly illustrates that the two approaches are complementary probes of the same underlying magnetic dynamics.

A minimal Heisenberg Hamiltonian for a bilayer $S=$ 1 system takes into account in-plane superexchange coupling $(J)$ between Ru moments $S$ as well as the intrabilayer 

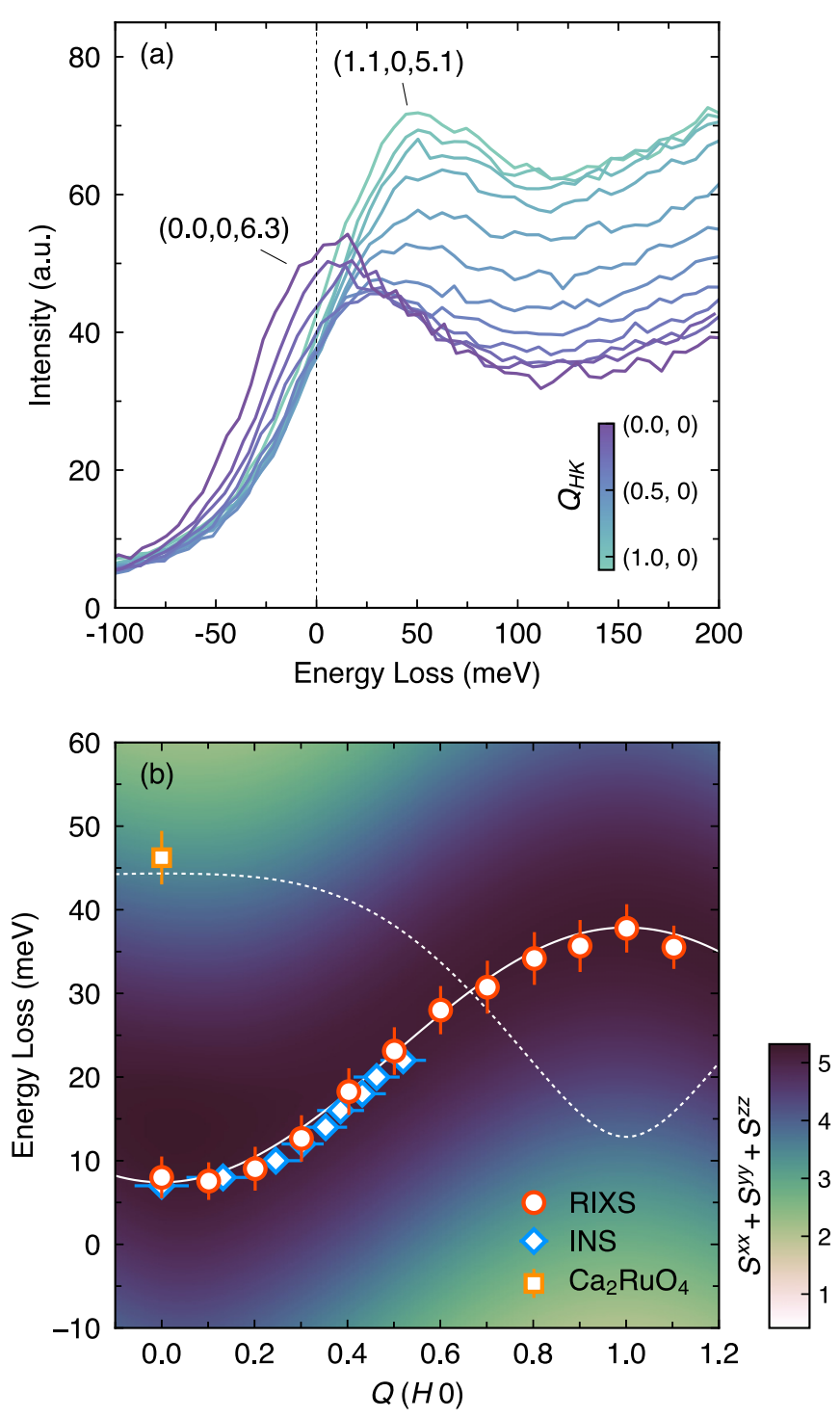

FIG. 3. (a) The momentum dependence of the RIXS signal below $E=200 \mathrm{meV}$ at $T=25 \mathrm{~K}$ from zone center to zone boundary shows the dispersion of the magnon. (b) The fitted magnon dispersion captured with RIXS corresponds well with inelastic neutron spectroscopy. Solid white lines plot the acoustic and optical modes of the bilayer spin-wave model as discussed in the text, while the color map represents the intensity calculated from the spin-spin correlation function that takes into account instrumental momentum and energy resolutions. The $\mathrm{Ca}_{2} \mathrm{RuO}_{4}$ spin-wave position determined from Fig. 2, and model spin wave based on Ref. [25] (dashed white line) are shown for comparison.

exchange interaction $\left(J_{c}\right)$ between directly adjacent moments stacked along the $c$ axis. Following prior work on $\mathrm{Ca}_{2} \mathrm{RuO}_{4}$ [25], we also introduce tetragonal $(E)$ and orthorhombic $(\epsilon)$ single-ion anisotropy (SIA) terms to account for the spin-wave gap,

$$
\begin{aligned}
H= & J \sum_{\langle i, j\rangle} \boldsymbol{S}_{i} \cdot \boldsymbol{S}_{j}+J_{c} \sum_{\langle i, j\rangle_{c}} \boldsymbol{S}_{i} \cdot \boldsymbol{S}_{j} \\
& +E \sum_{i} S_{i}^{z 2}+\epsilon \sum_{i} S_{i}^{x 2} .
\end{aligned}
$$

Coupling between bilayers, although important to the bulk antiferromagnetic response, is exceedingly weak and neglected here. Within the bilayer structure, we would expect to identify acoustic and optical spin-wave modes associated with the respective in-plane and intrabilayer couplings (see Supplemental Material [18]). The bilayer structure factor gives rise to an intensity modulation of the two modes, resulting in a maximum intensity of the in-plane (out-of-plane) mode at $Q_{\mathrm{L}}=5.0(7.5)$ and minimum at $Q_{\mathrm{L}}=7.5(5.0)$. As such, while the majority of the intensity is associated with the inplane mode, the energy-resolution limit makes it impossible to separate the two modes. We do, however, use this mixing to constrain the maximum energy of $J_{c}$. The primary spin wave for $J=-3.75 \mathrm{meV}, J_{c}=-6.5 \mathrm{meV}, E=5.5 \mathrm{meV}$, and $\epsilon=2.5 \mathrm{meV}$ is plotted in Fig. 3(b) as a white solid line, which corresponds well with parameters reported by Ke et al. [11]. The color map represents the calculated intensity of the excitation spectrum that takes into account the calculated IRIXS instrumental momentum and energy resolutions.

\section{Temperature and momentum dependence}

We now turn to detailed temperature and momentumtransfer RIXS studies. Figure 4(a) plots the low-energy spectra collected at $T=25,70$, and $300 \mathrm{~K}$ for $Q_{\mathrm{HKL}}=$ $(0,0,6.3)$, which are offset for clarity. Here, spectra were collected at $E_{i}=2839 \mathrm{eV}$ in order to enhance the visibility of the separate excitations that arise within the $t_{2 g}$ multiplet structure.

Above $T_{\mathrm{S}}$ and $T_{\mathrm{N}}$ at $70 \mathrm{~K}$, the $\sim 8 \mathrm{meV}$ magnon gap, marked by a dashed line in Fig. 4(a), closes as the signal intensity strongly increases. At $300 \mathrm{~K}$, ungapped magnetic excitations remain, although the signal is diminished and broadened. To capture the temperature evolution of the magnetic excitations through $T_{\mathrm{N}}$ and $T_{\mathrm{S}}$, a series of low-energy spectra were collected at $\Gamma$ from $T=35$ up to $65 \mathrm{~K}$. The magnon was modeled with a single pseudo-Voigt profile and the electronic continuum with a sigmoid function emerging from the elastic line. Two functions were used to fit the $t_{2 g}$ spectral weight. Following the observation in Fig. 1(g), we placed one peak around $600 \mathrm{meV}$ and the second one around $300 \mathrm{meV}$ [marked by vertical dashed lines in Fig. 4(a)]. A small profile around $100 \mathrm{meV}$ was also required to accurately model the excitation spectrum; such a feature was also seen in optical conductivity measurements (see Supplemental Material [18] and Ref. [9]). The resulting fit is shown in Fig. 4(a) for the $25 \mathrm{~K}$ dataset, with the magnon profile highlighted and other contributions plotted with dashed lines.

The magnitude of the fitted spin-wave gap and the integrated intensity of the magnetic fluctuations are plotted as a function of temperature in Fig. 4(b). The temperature dependency reveals that the base temperature $8(2) \mathrm{meV}$ magnon gap abruptly forms below the structural transition, $T_{\mathrm{S}}=48 \mathrm{~K}$, and not the Néel temperature, $T_{\mathrm{N}}=56 \mathrm{~K}$. The steplike behavior of the gap at $T_{\mathrm{S}}$, which we note coincides with the rotation of the spins from the $a$ axis to the $b$ axis [10], suggests that the magnetostructural transition arises with a marked change in the spin anisotropy of the system. At the same time, the increase in intensity of the magnon through this transition 

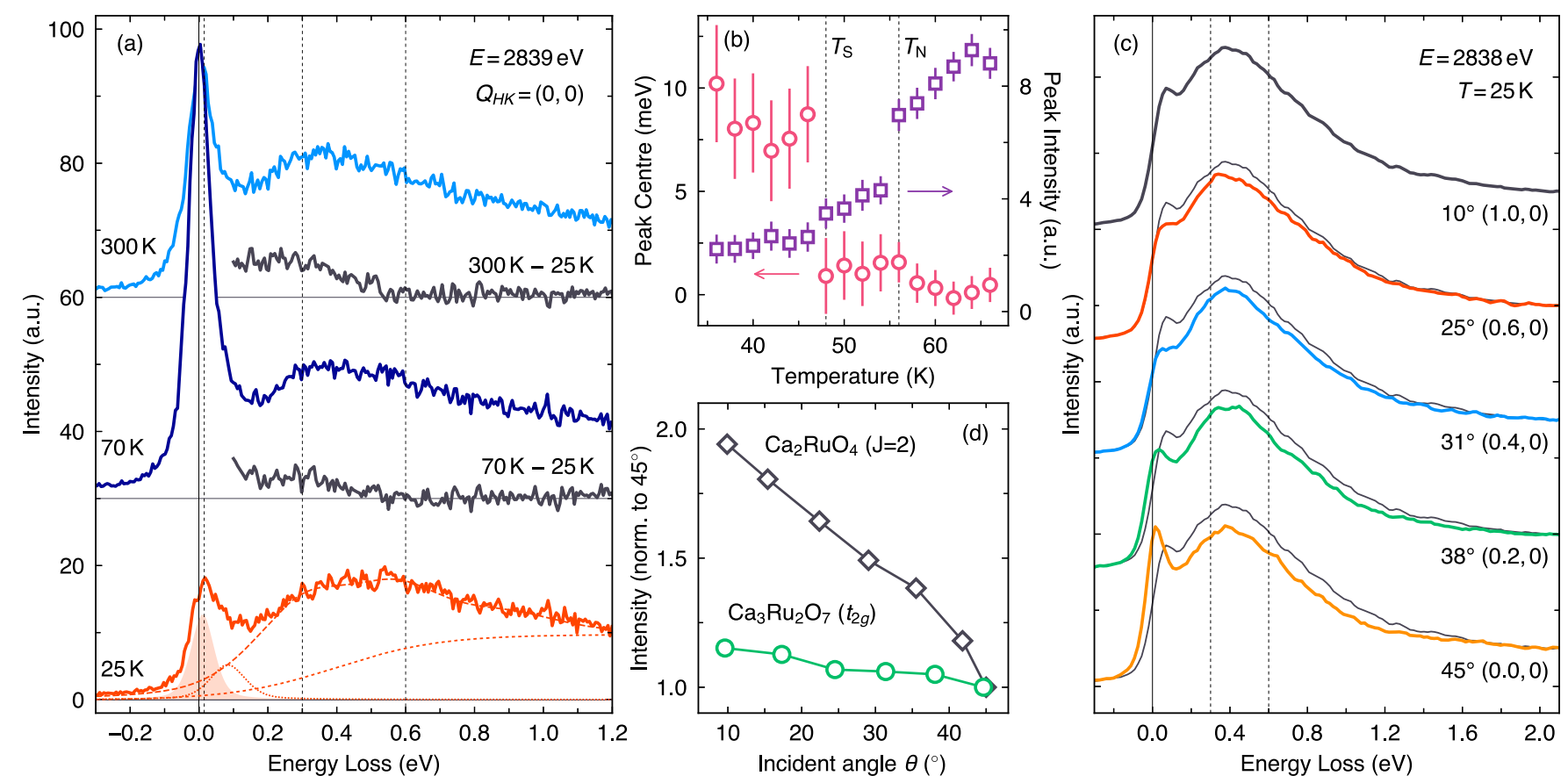

FIG. 4. (a) Temperature dependence of the magnon at the magnetic zone center, corresponding to the spin-wave gap. Above $T_{\mathrm{N}}$, the gap closes and strongly increases in intensity. At $T=300 \mathrm{~K}$, weak paramagnetic excitations remain. (b) Fitted magnitude and intensity of the spin-wave gap as a function of temperature. Results reveal that the gap closes at $T_{\mathrm{S}}$, while the bulk of the increase in intensity happens above $T_{\mathrm{N}}$. (c) Momentum dependence of the RIXS spectra at $T=25 \mathrm{~K}$ collected by varying $\theta$. Each spectrum was normalized to account for self-absorption and shifted vertically for clarity. Overlaid on each spectrum is the data collected at $\theta=10^{\circ}$. (d) Intensity of the $t_{2 g}$ features as a function of $\theta$ plotted in comparison to the spin-orbit $J=2$ excitation in $\mathrm{Ca}_{2} \mathrm{RuO}_{4}$. Only a moderate increase in spectral weight is observed in $\mathrm{Ca}_{3} \mathrm{Ru}_{2} \mathrm{O}_{7}$ at low $\theta$.

is gradual, as would be expected from a second-order phase transition.

A closer study of the temperature evolution of the electronic $t_{2 g}$ multiplet structure was conducted by subtracting the $25 \mathrm{~K}$ spectrum from the 70 and $300 \mathrm{~K}$ datasets, which are plotted in Fig. 4(a) as gray lines below the raw spectra. Here it can be seen that the increase in spectral weight extends up to around $0.5 \mathrm{eV}$, an energy scale that is much larger than the $<100 \mathrm{meV}$ pseudogap estimated from optical conductivity [9] and Raman scattering [28] measurements. It is therefore clear that components of the electronic structure evolve with temperature significantly away from the Fermi level.

Figure 4(c) plots the momentum dependence of the $\mathrm{Ca}_{3} \mathrm{Ru}_{2} \mathrm{O}_{7}$ RIXS spectrum at $T=25 \mathrm{~K}$ and $E_{i}=2838 \mathrm{eV}$, covering the $t_{2 g}$ multiplets and magnetic signal. The datasets were collected by varying the angle $\theta$ between the incoming photon polarization and the crystallographic $c$ axis [see Fig. 1(b)] to cover almost grazing incidence at $\theta=10^{\circ}$ to $\theta=45^{\circ}$. This range corresponds to changing the in-plane momentum transfer from $Q=(1,0)$ to $Q=(0,0)$. The spectra are normalized to a flat region around $2.0 \mathrm{eV}$ in order to simply account for the effects of self-absorption. The data at $Q=(1,0)$ are overlaid in gray with spectra collected at other incidence angles for comparison. Here it can be seen that while the magnon clearly disperses from $\Gamma$, the higher-energy signal associated with the $t_{2 g}$ excitations shows no measurable shift in spectral weight.

The lack of any clear dispersion implies that the origin of the excitations in the $t_{2 g}$ multiplet are local in nature and indeed arise from intraionic interactions. At the same time, the $t_{2 g}$ multiplet does undergo a small decrease in intensity as the incident angle is increased. This is in marked contrast to the polarization dependency of $\mathrm{Ca}_{2} \mathrm{RuO}_{4}$, which shows a very strong intensity variation of the $J=2$ excitation, effectively doubling between $\theta=45^{\circ}$ and $\theta=10^{\circ}$ [5]. This is a hallmark of the strong tetragonal distortion in $\mathrm{Ca}_{2} \mathrm{RuO}_{4}$, which drives the condensation of $J=1$ excitations. In Fig. 4(d), the integrated intensity of the $\mathrm{Ca}_{3} \mathrm{Ru}_{2} \mathrm{O}_{7}$ excitations between 0.2 and $0.6 \mathrm{eV}$ is plotted as a function of incidence angle and compared with the $\mathrm{Ca}_{2} \mathrm{RuO}_{4} \mathrm{~J}=2$ intensity. The intensity of the $\mathrm{Ca}_{3} \mathrm{Ru}_{2} \mathrm{O}_{7}$ signal is clearly devoid of such enhancement. Given that the magnitude of $\xi$ is unlikely to change by any significant amount between the two systems, it is clear that the crystal-field distortion is smaller in $\mathrm{Ca}_{3} \mathrm{RuO}_{7}$, and that the intraionic spin-orbit transitions strongly interact with the overwhelming electron-hole continuum.

\section{DISCUSSION}

Our experimental findings reveal two important features of $\mathrm{Ca}_{3} \mathrm{Ru}_{2} \mathrm{O}_{7}$. First, clearly dispersing ferromagnetic excitations were identified below $T_{\mathrm{S}}$, in excellent agreement with inelastic neutron scattering results. Second, unlike the sharp multiplets of $\mathrm{Ca}_{2} \mathrm{RuO}_{4}$, the RIXS spectra of $\mathrm{Ca}_{3} \mathrm{Ru}_{2} \mathrm{O}_{7}$ show a broad asymmetric feature with two energy scales-a metallic continuum response and excitations arising from damped ionic correlations in the $t_{2 g}$ band. 
The agreement between INS and RIXS validates our magnon observation and emphasizes the complementary nature of the two techniques. Although the energy resolution of INS is superior to RIXS, it lacks the count rates at higherenergy loss [see the zone-boundary data in Fig. 3(a)]. With this complete dataset at hand, we can with confidence report a magnon gap of $\sim 8 \mathrm{meV}$ and a zone-boundary energy of $\sim 37 \mathrm{meV}$ in $\mathrm{Ca}_{3} \mathrm{Ru}_{2} \mathrm{O}_{7}$. We observe that the nearest-neighbor exchange in $\mathrm{Ca}_{3} \mathrm{Ru}_{2} \mathrm{O}_{7}$ is ferromagnetic $(J=-3.75 \mathrm{meV})$ instead of AFM in $\mathrm{Ca}_{2} \mathrm{RuO}_{4}(5.8 \mathrm{meV})$, a hallmark of the correlated metallic state. In Fig. 3(b), we have included the peak position of the $\mathrm{Ca}_{2} \mathrm{RuO}_{4}$ magnon excitation at $\Gamma$ as determined from Fig. 2, as well as the model spin wave from Ref. [25] as a dashed white line, which clearly show the different magnetic dynamics of the two systems. More notable is the drastic reduction of the tetragonal SIA term $E=5.5 \mathrm{meV}$ when compared with $\mathrm{Ca}_{2} \mathrm{RuO}_{4}(22.75 \mathrm{meV})$. The primary driver behind the reduction in $E$ is likely the weaker $\Delta$ tetragonal crystal field in $\mathrm{Ca}_{3} \mathrm{Ru}_{2} \mathrm{O}_{7}$ due to reduced $\mathrm{RuO}_{6}$ distortions [10,24]. It is therefore of interest that the magnon gap appears only below $T_{\mathrm{S}}$ and not at $T_{\mathrm{N}}$, directly demonstrating the strong coupling between the structure and the magnetic moments. More specifically, these results show that the transition between the AFM- $a$ and AFM- $b$ states is associated with a marked change in spin anisotropy.

Our magnon results also give important insight into the field of RIXS studies of Ru-based compounds. In a recent $\mathrm{O}$ $K$-edge $\mathrm{RIXS}$ experiment on $\mathrm{Ca}_{3} \mathrm{Ru}_{2} \mathrm{O}_{7}$, the magnon mode we identify at the $\mathrm{Ru} L_{3}$-edge is not observed, despite the better energy resolution [29]. Although it is well known that single magnons are generally silent at the $\mathrm{O} K$-edge due to the lack of SOC in the $1 s$ core hole [30], special cases exist where this does not hold. A recent example includes the structurally related $5 d$-electron iridate systems $\mathrm{Sr}_{2} \mathrm{IrO}_{4}$ and $\mathrm{Sr}_{3} \mathrm{Ir}_{2} \mathrm{O}_{7}$, where both magnons and bimagnons could be probed thanks to the presence of strong SOC within the Ir $5 d t_{2 g}$ orbitals [31]. This suggests that even though $\mathrm{SOC}$ is present in the $\mathrm{Ru} 4 d t_{2 g}$ orbitals, it may not be strong enough to facilitate single spinflip excitations, leaving only bimagnons as an option at the $\mathrm{O}$ $K$-edge. In view of this observation, it is interesting to note that $\mathrm{O} K$-edge RIXS reports a feature at $55 \mathrm{meV}$ in $\mathrm{Ca}_{3} \mathrm{Ru}_{2} \mathrm{O}_{7}$ [29], a mode that we are not able to clearly identify at the $L_{3}$-edge, presumably due to the dominant magnon intensity.

We now move our discussion to the electronic excitations associated with the $t_{2 g}$ multiplet. It poses a challenge to extract the underlying structure in the RIXS spectrum of any metallic system due to the lack of sharp defining features. Here, however, via analysis of the incident-energy and temperaturedependency studies of the RIXS response of the system, we demonstrate that intra-atomic excitations survive in the metallic state. The comparison with $\mathrm{Ca}_{2} \mathrm{RuO}_{4}$ in Fig. 2 is then striking, as it shows that the RIXS spectra of $\mathrm{Ca}_{3} \mathrm{Ru}_{2} \mathrm{O}_{7}$, while heavily damped by the overlapping electron-hole continuum, nevertheless includes fingerprints of spin-orbit excitations and Hund's multiplets that are hallmarks of the ionic model. The ionic SOC $\xi$ and Hund's rule $J_{\mathrm{H}}$ terms that define these excitations are unlikely to be meaningfully different between the two systems. As such, these results indicate that the $\mathrm{Ca}_{3} \mathrm{Ru}_{2} \mathrm{O}_{7} t_{2 g}$ multiplet structure is reconfigured primarily by a smaller $\Delta$ term (which we note also drives the reduction of the tetragonal SIA spin-wave parameter). This observation is also supported by the optical conductivity, which remains featureless even at $300 \mathrm{meV}$ [9], excluding a simple band structure origin. At the same time, it is unclear why the spinorbit excitation would gain intensity in the paramagnetic state, as Fig. 4(a) shows. One possibility is that this is a consequence of a change in the electronic structure, although the energy scale here is much larger than reported in Raman and optical conductivity experiments $[9,28]$. Further investigation is needed to resolve this, including realistic dynamical meanfield theory (DMFT) calculations where local exchanges and band structure are treated equally.

\section{CONCLUSION}

We have reported upon extensive $\mathrm{Ru} L_{3}$-edge RIXS studies of the correlated multiband bilayer system $\mathrm{Ca}_{3} \mathrm{Ru}_{2} \mathrm{O}_{7}$. A well-defined magnetic excitation is observed, in excellent agreement with inelastic neutron scattering results, further forging an important link between these two experimental techniques. At higher energies, we discover a broad asymmetric $t_{2 g}$ excitation that is in stark contrast to the sharp multiplets in $\mathrm{Ca}_{2} \mathrm{RuO}_{4}$. However, the behavior of this feature in response to temperature, incident energy, and momentum transfer suggests that the vestiges of ionic multiplets remain present in the metallic state of $\mathrm{Ca}_{3} \mathrm{Ru}_{2} \mathrm{O}_{7}$, albeit heavily mixed with the electron-hole continuum. Our results show that RIXS at the $4 d$-electron $L$-edges is highly sensitive to the presence of on-site atomic interactions, providing an essential view into the interactions that underly correlated behavior in multiband metallic systems.

\section{ACKNOWLEDGMENTS}

We kindly thank A. Boris, K. Rabinovic, G. Khaliullin, and H. Liu for fruitful discussions. We thank L. Wang and K. Fürsich for assistance with the RIXS measurements, and J. Porras for assistance with the INS measurements. The project was supported by the European Research Council under Advanced Grant No. 669550 (Com4Com). We acknowledge DESY (Hamburg, Germany), a member of the Helmholtz Association HGF, for the provision of experimental facilities.
[1] E. Ohmichi, Y. Yoshida, S. I. Ikeda, N. Shirakawa, and T. Osada, Colossal magnetoresistance accompanying a structural transition in a highly two-dimensional metallic state of $\mathrm{Ca}_{3} \mathrm{Ru}_{2} \mathrm{O}_{7}$, Phys. Rev. B 70, 104414 (2004).
[2] F. Baumberger, N. J. C. Ingle, N. Kikugawa, M. A. Hossain, W. Meevasana, R. S. Perry, K. M. Shen, D. H. Lu, A. Damascelli, A. Rost, A. P. Mackenzie, Z. Hussain, and Z.-X. Shen, Nested Fermi Surface and Electronic Instability in $\mathrm{Ca}_{3} \mathrm{Ru}_{2} \mathrm{O}_{7}$, Phys. Rev. Lett. 96, 107601 (2006). 
[3] W. Bao, Z. Q. Mao, Z. Qu, and J. W. Lynn, Spin Valve Effect and Magnetoresistivity in Single Crystalline $\mathrm{Ca}_{3} \mathrm{Ru}_{2} \mathrm{O}_{7}$, Phys. Rev. Lett. 100, 247203 (2008).

[4] A. Georges, L. d. Medici, and J. Mravlje, Strong correlations from Hund's coupling, Annu. Rev. Condens. Matter Phys. 4, 137 (2013).

[5] H. Gretarsson, H. Suzuki, H. Kim, K. Ueda, M. Krautloher, B. J. Kim, H. Yavaş, G. Khaliullin, and B. Keimer, Observation of spin-orbit excitations and Hund's multiplets in $\mathrm{Ca}_{2} \mathrm{RuO}_{4}$, Phys. Rev. B 100, 045123 (2019).

[6] H. Suzuki, H. Liu, J. Bertinshaw, K. Ueda, H. Kim, S. Laha, D. Weber, Z. Yang, L. Wang, H. Takahashi, K. Fürsich, M. Minola, H.-C. Wille, B. V. Lotsch, B. J. Kim, H. Yavaş, M. Daghofer, J. Chaloupka, G. Khaliullin, H. Gretarsson, and B. Keimer, Quantifying the exchange interactions in the Kitaev Model system $\mathrm{RuCl}_{3}$ by the $\mathrm{Ru} \mathrm{L}$-edge resonant inelastic X-ray scattering, arXiv:2008.02037.

[7] H. Suzuki, H. Gretarsson, H. Ishikawa, K. Ueda, Z. Yang, H. Liu, H. Kim, D. Kukusta, A. Yaresko, M. Minola, J. A. Sears, S. Francoual, H.-C. Wille, J. Nuss, H. Takagi, B. J. Kim, G. Khaliullin, H. Yavaş, and B. Keimer, Spin waves and spin-state transitions in a ruthenate high-temperature antiferromagnet, Nat. Mater. 18, 563 (2019).

[8] Y. Yoshida, I. Nagai, S.-I. Ikeda, N. Shirakawa, M. Kosaka, and N. Môri, Quasi-two-dimensional metallic ground state of $\mathrm{Ca}_{3} \mathrm{Ru}_{2} \mathrm{O}_{7}$, Phys. Rev. B 69, 220411(R) (2004).

[9] J. S. Lee, S. J. Moon, B. J. Yang, J. Yu, U. Schade, Y. Yoshida, S.-I. Ikeda, and T. W. Noh, Pseudogap Dependence of the Optical Conductivity Spectra of $\mathrm{Ca}_{3} \mathrm{Ru}_{2} \mathrm{O}_{7}$ : A Possible Contribution of the Orbital Flip Excitation, Phys. Rev. Lett. 98, 097403 (2007).

[10] Y. Yoshida, S.-I. Ikeda, H. Matsuhata, N. Shirakawa, C. H. Lee, and $\mathrm{S}$. Katano, Crystal and magnetic structure of $\mathrm{Ca}_{3} \mathrm{Ru}_{2} \mathrm{O}_{7}$, Phys. Rev. B 72, 054412 (2005).

[11] X. Ke, T. Hong, J. Peng, S. E. Nagler, G. E. Granroth, M. D. Lumsden, and Z. Q. Mao, Spin-wave excitation in the antiferromagnetic bilayer ruthenate $\mathrm{Ca}_{3} \mathrm{Ru}_{2} \mathrm{O}_{7}$, Phys. Rev. B 84, 014422 (2011).

[12] B. Bohnenbuck, I. Zegkinoglou, J. Strempfer, C. SchüßlerLangeheine, C. S. Nelson, P. Leininger, H.-H. Wu, E. Schierle, J. C. Lang, G. Srajer, S. I. Ikeda, Y. Yoshida, K. Iwata, S. Katano, N. Kikugawa, and B. Keimer, Magnetic structure and orbital state of $\mathrm{Ca}_{3} \mathrm{Ru}_{2} \mathrm{O}_{7}$ investigated by resonant $\mathrm{x}$-ray diffraction, Phys. Rev. B 77, 224412 (2008).

[13] C. D. Dashwood, L. S. I. Veiga, Q. Faure, J. G. Vale, D. G. Porter, S. P. Collins, P. Manuel, D. D. Khalyavin, F. Orlandi, R. S. Perry, R. D. Johnson, and D. F. McMorrow, Spontaneous cycloidal order mediating a spin-reorientation transition in a polar metal, Phys. Rev. B 102, 180410(R) (2020).

[14] D. A. Sokolov, N. Kikugawa, T. Helm, H. Borrmann, U. Burkhardt, R. Cubitt, J. S. White, E. Ressouche, M. Bleuel, K. Kummer, A. P. Mackenzie, and U. K. Rößler, Metamagnetic texture in a polar antiferromagnet, Nat. Phys. 15, 671 (2019).

[15] S. Tsuda, N. Kikugawa, K. Sugii, S. Uji, S. Ueda, M. Nishio, and Y. Maeno, Mott transition extremely sensitive to impurities in $\mathrm{Ca}_{3} \mathrm{Ru}_{2} \mathrm{O}_{7}$ revealed by hard $\mathrm{x}$-ray photoemission studies, Phys. Rev. B 87, 241107(R) (2013).

[16] M. J. Krautloher, J. Bertinshaw, J. Porras, D. G. Joshi, G. Khaliullin, H. Trepka, M. H. D. Adroja, H. Walker, A. Ivanov, M. Enderle, U. Stuhr, B. J. Kim, and B. Keimer, Impurity induced localization of low-energy spin excitations in bilayer ruthenate $\mathrm{Ca}_{3} \mathrm{Ru}_{2} \mathrm{O}_{7}$ (unpublished).

[17] S. Nakatsuji and Y. Maeno, Synthesis and single-crystal growth of $\mathrm{Ca}_{2-x} \mathrm{Sr}_{x} \mathrm{RuO}_{4}$, J. Solid State Chem. 156, 26 (2001).

[18] See Supplemental Material at http://link.aps.org/supplemental/ 10.1103/PhysRevB.103.085108 for polarized light microscopy and INS spectra.

[19] H. Gretarsson, D. Ketenoglu, M. Harder, S. Mayer, F.-U. Dill, M. Spiwek, H. Schulte-Schrepping, M. Tischer, H.-C. Wille, B. Keimer, and H. Yavaş, IRIXS: A resonant inelastic X-ray scattering instrument dedicated to X-rays in the intermediate energy range, J. Synchrotron Rad. 27, 538 (2020).

[20] V. Bisogni, S. Catalano, R. J. Green, M. Gibert, R. Scherwitzl, Y. Huang, V. N. Strocov, P. Zubko, S. Balandeh, J.-M. Triscone, G. Sawatzky, and T. Schmitt, Ground-state oxygen holes and the metal- insulator transition in the negative charge-transfer rare-earth nickelates, Nat. Commun. 7, 13017 (2016).

[21] M. Minola, Y. Lu, Y. Y. Peng, G. Dellea, H. Gretarsson, M. W. Haverkort, Y. Ding, X. Sun, X. J. Zhou, D. C. Peets, L. Chauviere, P. Dosanjh, D. A. Bonn, R. Liang, A. Damascelli, M. Dantz, X. Lu, T. Schmitt, L. Braicovich, G. Ghiringhelli et al., Crossover from Collective to Incoherent Spin Excitations in Superconducting Cuprates Probed by Detuned Resonant Inelastic X-Ray Scattering, Phys. Rev. Lett. 119, 097001 (2017).

[22] K. Gilmore, J. Pelliciari, Y. Huang, J. J. Kas, M. Dantz, V. N. Strocov, S. Kasahara, Y. Matsuda, T. Das, T. Shibauchi, and T. Schmitt, Description of resonant inelastic x-ray scattering in correlated metals, arXiv:2011.04509.

[23] C. G. Fatuzzo, M. Dantz, S. Fatale, P. Olalde-Velasco, N. E. Shaik, B. Dalla Piazza, S. Toth, J. Pelliciari, R. Fittipaldi, A. Vecchione, N. Kikugawa, J. S. Brooks, H. M. Rønnow, M. Grioni, C. Rüegg, T. Schmitt, and J. Chang, Spin-orbit-induced orbital excitations in $\mathrm{Sr}_{2} \mathrm{RuO}_{4}$ and $\mathrm{Ca}_{2} \mathrm{RuO}_{4}$ : A resonant inelastic x-ray scattering study, Phys. Rev. B 91, 155104 (2015).

[24] M. Braden, G. André, S. Nakatsuji, and Y. Maeno, Crystal and magnetic structure of $\mathrm{Ca}_{2} \mathrm{RuO}_{4}$ : Magnetoelastic coupling and the metal-insulator transition, Phys. Rev. B 58, 847 (1998).

[25] A. Jain, M. Krautloher, J. Porras, G. H. Ryu, D. P. Chen, D. L. Abernathy, J. T. Park, A. Ivanov, J. Chaloupka, G. Khaliullin, B. Keimer, and B. J. Kim, Higgs mode and its decay in a twodimensional antiferromagnet, Nat. Phys. 13, 633 (2017).

[26] M. Kempa, B. Janousova, J. Saroun, P. Flores, M. Boehm, F. Demmel, and J. Kulda, The FlatCone multianalyzer setup for ILL's three-axis spectrometers, Physica B 385-386, 1080 (2006).

[27] J. Bertinshaw, M. J. Krautloher, J. Porras, A. Ivanov, B. J. Kim, and B. Keimer, Magnetic dispersion of $\mathrm{Ca}_{3} \mathrm{Ru}_{2} \mathrm{O}_{7}$ : Investigating the role of SOC in the $4 \mathrm{~d}$-electron ruthenates, Institut LaueLangevin (ILL) (2016), doi:10.5291/ILL-DATA.4-01-1495.

[28] H. L. Liu, S. Yoon, S. L. Cooper, G. Cao, and J. E. Crow, Raman-scattering study of the charge and spin dynamics of the layered ruthenium oxide $\mathrm{Ca}_{3} \mathrm{Ru}_{2} \mathrm{O}_{7}$, Phys. Rev. B 60, R6980 (1999).

[29] K. von Arx, F. Forte, M. Horio, V. Granata, Q. Wang, L. Das, Y. Sassa, R. Fittipaldi, C. G. Fatuzzo, O. Ivashko, Y. Tseng, E. Paris, A. Vecchione, T. Schmitt, M. Cuoco, and J. Chang, Resonant inelastic x-ray scattering study of $\mathrm{Ca}_{3} \mathrm{Ru}_{2} \mathrm{O}_{7}$, Phys. Rev. B 102, 235104 (2020). 
[30] V. Bisogni, L. Simonelli, L. J. P. Ament, F. Forte, M. Moretti Sala, M. Minola, S. Huotari, J. van den Brink, G. Ghiringhelli, N. B. Brookes, and L. Braicovich, Bimagnon studies in cuprates with resonant inelastic $\mathrm{x}$-ray scattering at the $\mathrm{O} K$ edge. I. Assessment on $\mathrm{La}_{2} \mathrm{CuO}_{4}$ and comparison with the excitation at $\mathrm{Cu} L_{3}$ and $\mathrm{Cu} K$ edges, Phys. Rev. B 85, 214527 (2012).
[31] X. Lu, P. Olalde-Velasco, Y. Huang, V. Bisogni, J. Pelliciari, S. Fatale, M. Dantz, J. G. Vale, E. C. Hunter, J. Chang, V. N. Strocov, R. S. Perry, M. Grioni, D. F. McMorrow, H. M. Rønnow, and T. Schmitt, Dispersive magnetic and electronic excitations in iridate perovskites probed by oxygen $K$-edge resonant inelastic x-ray scattering, Phys. Rev. B 97, 041102(R) (2018). 